\title{
American Spinal Injury Association Abstracts of Meeting: 1985
}

\author{
Held in Atlanta, Georgia, U.S.A. April 15-17, 1985.
}

\section{The Neurological Consequences of Post-Traumatic Neural Canal Impingement \\ J. S. Keene, M.D., S. P. Fischer, M.D., D. S. Drummond, M.D. and P. A. Turski, M.D. \\ Division of Orthopedic Surgery and Department of Radiology, University of Wiscon- sin Clinical Science Centre, Madison, Wisconsin, U.S.A.}

The authors studied 80 consecutive patients who had Computerized Tomography (CT) evaluation of acute, traumatic thoracolumbar fractures and correlated the amount of canal compromise with each patient's: 1) neurological status; 2) level of injury; 3) type of fracture; and 4) latent signs of spinal stenosis. Standard radiographs and CT scans were evaluated to determine the type of fracture and level of injury. Injuries were classified in one of four major categories of fractures and the patient's neurological status was classified according to the standards of the American Spinal Injury Association. Neural canal compromise was determined from CT scans that were obtained at the level of injury prior to operative reduction or non-operative treatment. Patients who had no neurological lesion at the time of their initial injury were subsequently evaluated for latent spinal stenosis by telephone survey. Of the 80 patients studied, 17 had complete lesions, 17 had incomplete lesions, and 46 had no deficits. The average canal compromise for these three neurological categories was $62 \%, 55 \%$ and $27 \%$ respectively. Although the average percent compromise was significantly higher in patients with complete and incomplete lesions, the range of percent compromise was the same for all three neurological groups. Average compromise for each level of injury was thoracic $29 \%$ (range $0-100$ ), thoracolumbar $37 \%$ (range $0-93$ ) and lumbar 58\% (range $0-100$ ). The average compromise was significantly higher in the lumbar spine but the percent of patients with neural deficit was significantly higher in the thoracic spine. The percent of patients with neural deficits in the thoracic and lumbar spine was 58 and 27 percent respectively.

The average percent compromise of the canal and the neurological deficit with each type of fracture were as follows: for flexion-distration fractures, the average percent compromise was 36\% (range 0-93\%), for burst fractures $52 \%$ (range $0-100^{\circ}$ ), for wedge compression $0 \%$, and for fracture-dislocations $46 \%$ (range $\left.0-100^{\circ}{ }_{0}\right)$. The average compromise was significantly higher in burst fractures and fracture-dislocations compared to flexion-distraction injuries and wedgecompression fractures, but the neurological deficits did not correlate with the average canal compromise associated with each type of injury. Although burst fractures produced the highest average canal compromise, only $31 \%$ of these patients had a neurological lesion compared to $46 \%$ with flexion-distraction 
injuries and $79 \%$ with fracture-dislocations. Similarly one of the eight patients with a wedge compression fracture had a neurological deficit yet the canal compromise for this patient was $0 \%$.

Forty-six patients had no neurological deficit. Twenty-nine of these were contacted by telephone to determine if they had signs or symptoms of latent spinal stenosis. (The remaining 17 patients could not be located.) All 29 reported no signs or symptoms of spinal stenosis at an average of three years follow up.

The authors feel this study confirmed that the neural canal compromise caused by bony encroachment of the canal does not correlate with the neurological status of the patient. It was felt that the neurological sequelae of fractures and dislocations of thoracic and lumbar spine appear to be more related to the level of injury and the type of fracture than percent of compromise of neural canal. This suggested that the neurological deficit is the result of trauma occurring at the time of injury rather than the percent of post-traumatic bony encroachment documented by CT scans.

\section{Lumbosacral Evoked Potentials (LSEP) and Cortical Somatosensory Evoked Potentials (SEP) in Patients with a Cauda Equina Lesion}

L. Don Lehmkuhl, Ph.D., Milan R. Dimitrijevic, M.D., DSc and Janez Zidar, M.D.

Department of Clinical Neurophysiology, The Institute for Rehabilitation and Research, Department of Rehabilitation, Baylor College of Medicine, Houston, Texas 77030, U.S.A.

The study was undertaken to compare how well the lumbar evoked potential and somatosensory evoked potential correlated with the clinical status of the patient and to define characteristics of the lumbosacral evoked potentials and the somatosensory evoked potential in patients with clinical electromyographical signs of cauda equina lesions. Patients were divided into three groups.

Group A were patients with cauda equina lesions only, Group B were patients with cauda equina and conus medullaris lesions, Group $C$ were patients with cauda equina lesions in addition to cervical or high thoracic cord injury. A healthy group served as controls.

The study showed that measurement of lumbosacral evoked potentials in patients with clinical symptoms of cauda equina, conus medullaris lesions can provide additional evidence to help delineate the extent of the lesion. In these patients, each major component of the lumbosacral evoked potential could be absent or the peak could have a reduced amplitude or prolonged latency. The degree of impairment of the lumbosacral evoked potential and somatosensory evoked potential ran parallel to the degree of severity of the cauda equina lesion. The recording of the lumbosacral evoked potentials and somatosensory evoked potential responses with surface electrodes represents a reliable test for the detection of mild cauda equina abnormalities and for providing subclinical evidence of limited sparing in severe cauda equina lesions. Patients with spinal cord injury of traumatic origin localized at the cervical or thoracic vertebral levels may also have an unrecognized lesion of the cauda equina which can cause symptoms that are viewed as unusual until the association with the cauda equina lesion is recognized. 


\section{Cervical Spinal Cord Injury and Ankylosing Spondylitis}

Dominic Foo, M.D., Mehdi Sarkarati, M.D. and Victoria Marcelino, M.D. Spinal Cord Injury and Neurologic Services, West Roxbury Veterans Administration Medical Center, 1400 VFW Parkway, Boston, Massachusetts 02132, U.S.A.

The authors identified seven patients with cervical spinal cord injury and ankylosing spondylitis. Six were male and one was female with ages ranging from 60 to 81 years. Six patients sustained their injury due to a fall and one in an automobile accident. History of alcohol consumption preceding the accident was present in five patients. Levels of injury were as follows: C3-4 in one patient, C5-6 in one patient, C6-7 in three patients. One patient had no spinal bony injury and in another the nature of the spinal injury was unknown. The mechanism of injury was hyperextension in four patients, probably hyperextension in two and flexion in one. Neurological examinations on admission revealed one to be sensory and motor incomplete, one sensory complete but motor incomplete, and sensory and motor complete in five. In four patients there was a history of neurological deterioration before admission. Three patients were initially treated with a cervical collar and four underwent skull traction. Significant neurological recovery was seen in one patient but four had slight or no improvement. Two others experienced further neurological loss while in skull traction. Operative reduction and fixation of the fractured spine was performed in two patients with no postoperative improvement. In one an epidural haematoma was found.

Three of these patients died from medical complications and one expired as a result of an ascending cervical cord lesion. Autopsy studies in one patient showed two separate spinal cord lesions in the presence of only one spinal fracture and in another extensive intraspinal callus formation at the fracture site causing marked constriction of the spinal cord.

The conclusions were that most of the patients had a history of alcoholic consumption prior to their injury and about half of these patients had a history of neurological progression before they were seen in the emergency room. They concluded that operative treatment of the unstable fracture was disappointing in reversing neurological deficits and likewise skull traction had been unsatisfactory in two of four patients who experienced further neurological loss while under this treatment.

\section{Neurological and Skeletal Outcomes in Patients with Closed Cervical Spinal Cord Injury}

William H. Donovan, M.D., Dennis R. Kopanicky, M.D., Ewelina Stolzmann, M.D. and R. Edward Carter, M.D.

The Institute for Rehabilitation and Research, Houston, Texas 77030, U.S.A.

The authors have sought to add information to the issue regarding operative versus non-operative management of spinal cord injury by measuring specific neurological and skeletal outcomes in both surgically and non-surgically treated spinal cord injured patients since 1981. All these patients were treated within the same system of care and were followed within this system from the date of their injury until at least one year. 
The outcomes were evaluated at 3, 6 and 12 months post injury and included A) neurological level and degrees of completeness of injury per Frankel's criteria; B) the mechanism of injury modified from Braakman and Penning, namely 1 . extension, 2. compression, 3. flexion with compression, and 4. flexion with distraction; C) the degree of angulation; D) displacement distance in millimetres; E) the presence of callus anteriorly; F) the presence or absence of instability, defined as abnormal movement of the cervical spine in or around the area of injury. Eighty patients qualified for this study over a two year period from July 1981 through June 1983. Nineteen were excluded because they had penetrating rather than closed injuries or fractures of $\mathrm{C} 1$ or $\mathrm{C} 2$ only, or no bony injury, or expired, or had been lost to follow up. Fifty-two males and nine females with a mean age of 30 years remained. Seventeen of the 61 patients underwent fusion for spinal stabilization, 4 anterior, 13 posterior, while one had an early laminectomy for decompression. Forty-three had no surgery.

Of the operated patients, ten $(55 \%)$ improved neurologically by at least one Frankel grade. Seven $\left(39^{\circ},{ }_{0}\right)$ remained unchanged and one $(6 \%)$ was worse. Of the non-operative patients, $31\left(72^{\circ}\right.$ o $)$ improved, $11(26 \%)$ remained the same, while $1(2 \%)$ was worse. None of the surgical group demonstrated instability at any time. Similarly none of the non-operative patients whose mechanism of injury was due to extension, compression or flexion with compression showed late instability. Only those patients who were injured by flexion with distraction, that is who demonstrated disruption of the posterior ligaments, were at risk for this type of complication. Three $\left(33^{\circ} \circ\right)$ of the nine patients injured in this way developed late instability.

The authors concluded that 1 ) recovery of neurological function may proceed over a 12 month period in many patients with cervical cord injury including those where the initial evaluation revealed a complete injury. 2) excluding one patient whose angulation was uncorrected and worsened by laminectomy in the surgical group, alignment was more anatomical. Angulation was generally acceptable in the non-surgical groups since the greatest angulation was 35 degrees and only two were worse than 25 degrees. Displacement was about the same in both groups and did not exceed eleven millimetres in either group. 3) Most patients formed callus anterior to the injured vertebral bodies, regardless of the mechanism of injury and regardless of the method of treatment. 4) Most patients managed conservatively will be stable after three months. Only those with flexion distraction injuries appeared to be at risk of late instability. 5) A large percentage of patients treated conservatively or with posterior fusion who had anterior reactive bone formation would suggest that when surgery is considered for stabilization, posterior fusion may be the surgical approach of choice in a large number of patients. A large number of these patients will not only have the posterior fusion but also the anterior reactive bone formation so that a 'two column' fusion results. 


\section{Anterior Decompression and Rigid Internal Fixation of Cervical Spine Injuries}

Isadore G. Yablon, M.D., Joe Ordia, M.D., Murray Freed, M.D., Edward Spatz, M.D. and Ronald Mortara, M.D.

Department of Orthopedic Surgery, Boston University Medical Center, 75 East Newton Street, Boston, Massachusetts 02118, U.S.A.

The authors described a study to determine the efficacy of rigid anterior stabilization of the cervical spine following decompression for burst fractures and extension injuries. Nineteen patients were evaluated after operation and were followed for two months to three years. Fifteen had burst fractures and four had extension type injuries, all of which were determined to be unstable. The surgical procedure described was the standard approach with excision of the disk above and below the injured vertebral body and removal of the vertebral body. If the anterior longitudinal ligament was intact, the spinal cord was not explored. However, if the anterior longitudinal ligament was torn then exploration was carried out in order to remove any loose fragments. After the end plates of the vertebra above and below were freshened down to bleeding bone, a $\frac{1}{4}{ }^{\prime \prime}$ drill hole was made through the centre of the vertebral body above and below the fracture. The height of the vertebral body was measured and a drill stop used on the drill bit to assure that penetration of the vertebral body does not occur. This hole was then packed with methyl methacrylate. A $3.2 \mathrm{~mm}$ drill bit is then used to drill a hole in the cement. The holes were tapped and a bicortical full thickness graft obtained from the anterior crest of the ilium. This graft was cut to size and inserted under compression between the two vertebrae. A special three-hole $3 \mathrm{~mm}$ thickness plate is then applied and secured with three cortical screws. The middle screw enters the graft and prevents migration of the graft. For extension injuries, it was necessary to mobilise two vertebrae above and two vertebrae below the level of dislocation and the technique for inserting the screws was described.

Postoperatively, patients wear a soft collar for approximately ten days and then the collar was discontinued. There were no instances of loosening of fixation. There was one case of recurrent laryngeal nerve paralysis. Transient dysphagia was common but generally subsided within five days. Forty-two percent of the patients had an increase of one level of improvement within one year and $22 \%$ had improvement of two levels at one year. The authors feel that the results show that rigid internal fixation of burst fractures and extension injuries is possible and that thorough decompression allows for a greater degree of recovery.

Interspinous Segmental Wiring of Thoracolumbar Fractures: The Wisconsin System

J. S. Keene, M.D., S. P. Schemmel, M.D., B. Adamsom, M.D., A. L. Breed, M.D., D. S. Drummond, M.D. and D. L. Wackwitz, M.D.

Division of Orthopedic Surgery, University of Wisconsin Clinical Science Center, 600 Highland Avenue, Madison, Wisconsin 53792, U.S.A.

Recently, the use of segmental wires to augment the instrumentation utilised to stabilise fractures of the thoracic and thoracolumbar spine has been advocated. 
However, sublaminar segmental wiring has been associated with a $10-16 \%$ incidence of neurological complications. In an effort to provide the benefits described for sublaminar segmental wiring without the added risk of passing the wires, the authors became interested in utilising the base of the spinous process for segmental spinal instrumentation. Their study reported: 1) testing of interspinous segmental spinal instrumentation on a bovine fracture model; and 2) the preliminary clinical results of 25 patients with thoracolumbar fractures that had interspinous segmental wiring in addition to distration and/or compression instrumentation. In the laboratory, three methods of spinal instrumentation for stabilising simulated burst fractures in ten bovine spines were tested. The three methods were 1) bilateral distraction instrumentation; 2) bilateral distraction instrumentation augmented with sublaminar wires; and 3) bilateral distraction instrumentation augmented with wires secured to the base of the spinous process. The instrumented spines were tested in forward flexion, lateral flexion, and axial rotation.

The two methods of spinal wiring reduced displacement in all planes tested over bilateral distraction rods alone. For forward flexion, interspinous wiring reduced displacement by $16^{\circ}{ }_{0}$ and sublaminar wiring reduced displacement by $46 \%$. In lateral flexion, displacement was reduced by $32 \%$ by interspinous wiring and $33^{\circ} \circ$ by sublaminar wiring. In axial rotation displacement was reduced $31 \%$ by interspinous wiring and $59{ }^{\circ}{ }_{0}$ by sublaminar wiring. The Wisconsin system (interspinous process wiring) was most effective against lateral flexion and rotational loads.

Twenty-five patients with unstable thoracolumbar fractures that were treated with Harringron distraction and/or compression rods supplemented with interspinous segmental instrumentation were compared with 30 patients with the same fractures who were treated with the same instrumentation but without interspinous wiring.

The average percent correction achieved in anterior compression immediately postoperatively was the same for wired and non-wired groups. Similarly there was no statistically significant difference in loss of correction for either system from immediate postoperative to one year follow up. The average percent correction obtained for displacement was similar for the wired and non-wired spines. At one year there was no significant difference between the wired and non-wired spines in maintenance of correction of displacement. The wired group lost only $2^{\circ}{ }_{o}$ and the non-wired group only $9 \%$ of the initial corrections achieved. However, the two systems showed significantly different results with regard to maintenance of correction of angle deformity. Although the immediate postoperative correction of angle deformity showed no difference for the two systems, at one year the wired group lost only $18^{\circ}$ of their correction whereas the non-wired group had lost $24^{\circ}{ }_{0}$ of their correction. There was also a greater number of hook site failures in the non-wired patients.

In summary, laboratory testing of the Wisconsin System of interspinous segmental instrumentation shows that it provided increased stability over distraction rods alone, particularly versus lateral flexion and rotational loads. Clinically, the system produced an improvement in the loss of correction of angle of deformity and there were fewer hook site failures than was observed in the non-wired group. There were no neurological deficits attributed to the 
interspinous wiring and the fusion rate was $100 \%$ in the 25 patients reviewed at one year.

\section{The State of the National SCI Database}

P. R. Fine, Ph.D., M. J. DeVivo, Dr. P.H., B. K. Go, B.A., P. B. Lazarus, P. L. Kartus, M.P.H., R. D. Rutt, B.S. and S. L. Stover, M.D.

National Spinal Cord Injury Statistical Center, The University of Alabama at Birmingham Spinal Cord Injury Care System, The Spain Rehabilitation Center, University Station, Birmingham, Alabama 35294, U.S.A.

The National Spinal Cord Injury Statistical Center (NSCISC), formerly the UAB Spinal Cord Injury Data Management Service, was established at the University of Alabama at Birmingham in March, 1983 as the federally designated successor to the Phoenix, Arizona based National Spinal Cord Injury Data Research Center (NSCIDRC).

As of February, 1985, initial hospitalization and follow up data on over 9000 patients constituted the National Spinal Cord Injury Statistical Center Database which served as the focus of this study.

Inspection and analysis of the database revealed that the proportion of patients admitted to the several federally-sponsored SCI Care Systems has increased from $3 \%$ (of the nationally estimated incidence) in 1973 to over $14 \%$ in 1984 . This increase is due, in part, to a slight increase in the number of SCI Care Systems as well as improved rates of capture witnessed in many Systems.

Inspection and analysis of the database showed that the mean age at injury had increased slightly, from a previously reported 29 years to the current 31 years. Eighty-two percent $\left(82^{\circ}\right)$ of all SCI patients were male; a $4: 1$ male to female ratio that has been constant since 1973. Nationally, the four leading causes of SCI are motor vehicle crashes $(46.7 \%)$; falls $(20.4 \%)$, sporting accidents $\left(14.5^{\circ}{ }_{0}\right)$, and acts of violence-primarily gunshot wounds $(14.3 \%)$.

The proportion of patients admitted with cervical cord lesions $(54 \%)$ has remained fairly constant since the mid 70's although the proportion admitted with neurologically complete lesions has statistically declined from $62 \%$ to $46 \%$. It is believed that this decline in the proportion of neurologically complete lesions is due, in large part, to improved emergency medical services provided by well trained, highly skilled EMS personnel at the injury site.

Following completion of rehabilitation, nearly $90 \%$ were discharged to their usual, pre-injury place of residence, with less than $4 \%$ being discharged to nursing homes.

While $59.2 \%$ of all SCI patients were reported to have been employed at the time of injury, only $9.4 \%$ were employed one year post-injury. However, this post-injury employment figure had increased to $37.1 \%$ within eleven years.

Days from injury to System admission increased slightly (20 to 24) between 1973 and 1975. However, in 1976 the average number of days between injury and System admission began to decline so that at present, the average time between injury and admission is only nine days. For all SCI patients, the average length of stay is 99 days. As might be expected, neurologically complete tetraplegic patients have longer average lengths of stay (132 days) than do any other category of patients. Of even greater importance is the fact that there is a 
definite, downward trend in the lengths of stay for every neurological category.

Despite the fact there has been a statistically significant reduction in the average length of stay, costs associated with medical care continue to rise at a rate which consistently exceeds the inflation rate. Mean system charges adjusted to 1984 dollars were noted to have increased from $\$ 36,941$ in 1973 to $\$ 55,624$ in 1982. However, a slight decline to $\$ 48,992$ was documented in 1984 .

Pressure sores were the most frequently reported predischarge complication, occurring in approximately $24 \%$ of all SCI patients. However, the most severe Grade IV pressure sore were rare, developing in only $1 \%$ of the patients. The incidence of bladder stones appears to have declined from $10 \%$ to approximately $5 \%$ before discharge. This apparent reduction in the incidence of bladder stones is believed to be due, in part, to increased utilisation of intermittent catheterisation as the bladder management technique of choice.

The 'Cumulative Ten Year Survival Rate' for SCI patients was determined to be $86.1 \%$ compared with a $98.5 \%$ survival rate for a cohort of 24 year old non spinal cord injury patients. The risk of dying was greatest during the first post injury year $(4.42 \%)$; however, this risk declined to only $0.44 \%$ by the tenth post injury year. In this series, respiratory complications (primarily pneumonia) were the leading cause of death, followed by unexplained cardiac arrest, subsequent accidents and suicides, heart disease, pulmonary emboli, and septicaemia, Renal disease, formerly the leading cause of death among SCI patients accounted for only $3 \%$ of all reported deaths.

\section{Predicting Lengths-of-Stay for Spinal Cord Injury Patients}

P. R. Fine, Ph.D., M. J. DevVivo, Dr. PH., B. K. Go, B.A., P. B. Lazarus, P. L. Kartus, M.P.H., R. D. Rutt, B. S. and S. L. Stover, M.D.

National Spinal Cord Injury Statistical Center, University of Alabama at Birmingham Spinal Cord Injury Statistical Center, The Spain Rehabilitation Center, University Station, Birmingham, Alabama 35294, U.S.A.

To assist federal officials establish equitable and realistic reimbursement guidelines for newly injured SCI patients, the American Spinal Injury Association (ASIA) commissioned the National Spinal Cord Injury Statistical Center (NSCISC) to study lengths-of-stay (LOS) in SCI patients.

The study population consisted of 2189 patients who were: (1) injured between 1973 and 1984; (2) admitted to any of 18 federally-sponsored regional spinal cord injury care systems within 24 hours of injury, and (3) ultimately discharged alive. Variables judges capable of influencing LOS on a priori basis included: age at injury, sex, neurological level of spinal cord lesion, Frankel Grade, number of pre-specified/pre-existing medical conditions, number of pre-specified associated injuries, number of pre-specified medical complications developing during initial hospitalisation, number of pre-specified surgical procedures performed during initial hospitalisation, worst pressure sore grade and ventilator dependency during initial hospitalisation.

Multiple linear regression was used to develop a predictive model for overall LOS. Using the predicted LOS, the study population was divided into seven groups based on numeris cut points associated with severity of injury. Results were validated with a second study population consisting of 196 patients 
discharged after completion of the original study. Three variables, age at injury, sex and pre-existing medical conditions did not contribute significantly to the predictive model and were deleted.

Actual mean LOS ranged from 11 days in Group 1 patients (those without residual neurological deficit and an uneventful course of recovery) to 229 days in Group 7 patients (those with residual neurological deficits and numerous medical complications, surgical procedures, etc.). Coefficients of variation were small suggesting that the seven groups were relatively homogeneous with respect to overall LOS.

Actual mean costs of care ranged from $\$ 5149$ in Group 1 to $\$ 141970$ in Group 7. Again, the coefficients of variation were small suggesting the groups were relatively homogeneous with respect to overall costs of care.

Only 73 patients in the study population were Medicare beneficiaries. Based upon closer inspection of their data it was determined that in general, Medicare beneficiaries have slightly lower lengths of stay and costs of care than the total study population.

Because the predictive model appears to have identified seven, valid, homogeneous groups, it is recommended that future SCI patients be categorised according to the LOS calculation method devised by NSCISC workers and that prospective reimbursement be based upon the targets established in this study.

\section{Peer Support Programme-Method and Outcome Analysis}

Cindy Burns, M.Ed. and Jill S. Glass, R.P.T., M.Ed.

Shepherd Spinal Center, 2020 Peachtree Road, N.W. Atlanta, Georgia 30309, U.S.A.

Peer support is defined as 'help provided by a disabled person who has attained disability-related experiences, knowledge and coping skills and helps other disabled people and their significant others to cope with disability related experiences.' Peer support programme is utilised at the Shepherd Spinal Center as an adjunct to other services offered in the rehabilitation programme. In order for an individual to qualify as a peer supporter, the individual has to be at least six months post discharge from a rehabilitation/hospital programme. Family members of individuals with spinal cord injury can also apply as peer supporters. The peer supporters, after careful evaluation of their application form and subsequent interview, are carefully selected assessing the individual's potential to be a role model for newly injured patients. A mandatory training programme then follows. It is offered on an introductory and advanced basis with the programme consisting of experiential role playing exercise, enacting common situations which peer supporters might encounter in meeting with patients. The advanced training session is repeated yearly and is mandatory for those wishing to continue in the programme.

The peer support committee tries to match peer supporters with those requiring help for similarities of backgrounds, interests and injury. An evaluation of the programme indicated $98 \%$ of the patients reported it was helpful to talk to individuals with spinal cord injury who have been out in the 'real world' and that seeing well adjusted individuals with similar injuries had a positive impact on their confidence level and motivation. The peer supporters themselves felt 
useful and enjoyed sharing disability related experiences with newly injured individuals and offering support and advice. Based on these reviews, it was felt that peer support is a valuable resource to individuals with physical disability.

\section{Advances in Paraplegic Walking Using Electrical Stimulation}

E. B. Marsolais, M.D., Ph.D., Rude Kobetic, M.S., Howard Chizeck, Sc.D. and Ronald Lew, M.D.

Veterans Administration Medical Center, Cleveland, Ohio. Case Western Reserve University, Cleveland, Ohio. Veterans Administration Medical Center, Research, Room K205, 10701 East Boulevard, Cleveland, Ohio 44106, U.S.A.

Six patients with complete spinal cord lesions from T4 to T11 and no significant peripheral nerve damage were implanted bilaterally with percutaneous intramuscular electrodes in the hip flexors, extensors and abductors, knee extensors, dorsi and plantar flexors. Results show that implantation of the hamstrings, gluteals and soleus in addition to hip flexors, knee extensors and dorsiflexors allowed better posture control and improved quality and speed of gait. With practice, patients progressed from using parallel bars to a rolling walker and in two cases to axillary crutches. Three subjects were able to climb stairs with bilateral railings, and one required some assistance descending.

Although the authors demonstrated improvements in the functional electrical stimulation system for walking in paraplegic persons, they felt that many problems still remain to be solved. Even the best subjects did not achieve what could be defined as functional walking. Problems preventing full realization at this time include variable muscle response time, muscle fatigue, and inadequate muscle strength in some of the implanted muscles. Implementation of a Functional Neuromuscular Stimulation System
for a Tetraplegic Upper Extremity

M. W. Keith, M.D., P. H. Peckham, Ph.D., A. A. Freehafer, M.D., G. B. Thorpe, R. E. Gordon, OT/L, M. Sobel

Case-Western Reserve University, 3395 Scranton Road, Cleveland, Ohio 44109, U.S.A.

The authors described a six year experience to develop a routinely available functional neuromuscular stimulation system providing lateral prehension and release or palmar prehension and release in over 20 patients. The patients were selected from among C5 and C6 patients who were free from active infection and who had a sufficient complement of upper motor neuron innervated muscles in the hand and forearm.

Using techniques of surface and transcutaneous electrical mapping, the exact neurological lesion was determined. Muscles were classified as voluntary, lower motor neuron, or upper motor neuron based on their manual muscle test, electrical inexcitability, or response to transcutaneous or percutaneous electrical stimulation. All patients showed sufficient upper motor neuron innervated muscles to permit the design of a stimulation system. Static wrist hand orthosis was used to replace the wrist extensors and reduce the number of joints to be stabilized. No surgical procedures other than thumb interphalangeal joint 
stabilization and posterior deltoid transfers for elbow extension were performed. Flexor digitorum sublimis was stimulated in 22 patients, flexor digitorum profundus in 17 patients, sometimes in combination in order to provide index finger flexion. Finger extension was obtained in 13 patients by stimulating the extensor digitorum communis. The flexor pollicis longus was stimulated in four patients. The thumb extensor was stimulated in 23 patients reflecting the importance of extensor pollicis longus to control thumb position in palmar abduction or key grip. If the extensor pollicis longus was not suitably strong, the extensor pollicis brevis was substituted. In 11 patients, the median intrinsic muscles, abductor pollicis brevis and opponens were stimulated as the techniques were refined in this series for palmar prehension. All 21 patients in the study were given access to lateral pinch prehension as the initial gripping pattern. Only one patient had a suitable wrist extensor for electrical stimulation indicating the characteristic lesion found in C5 tetraplegia. The authors felt that functional neuromuscular stimulation remains a potentially important clinical tool for rehabilitation of the patient with a high level cervical spine injury where tendon transfer surgery for hand grip is impossible.

\section{The Value of Computerised Closed-Loop Functional Electrical Stimulation in Spinal Cord Injury Patients}

Barth A. Green, M.D., Myron Goldberd, Ph.D. and Bernard Brucker, Ph.D. University of Miami School of Medicine, 1501 Northwest 9th Avenue, Miami, Florida 33136, U.S.A.

The clinical laboratory at the University of Miami School of Medicine evaluated the effects of computerized closed-loop functional electrical stimulation in approximately 20 patients with complete and incomplete spinal cord injuries over 24 months. A closed-loop FES leg trainer was tested on a group of complete paraplegics and tetraplegics using quadricep stimulation of one leg with the muscle mass, serum and urine chemistry, stress EKGs, echocardiography, and one study included biopsies. The significant results were noted in reversal of muscle atrophy. The same functional electrical stimulation system was applied to patients with incomplete lesions when specific muscle group strengthening was desirable. Open loop functional electrical stimulation was also applied to the upper extremities of tetraplegic patients in conjunction with computerised multi-channel EMG biofeedback. Also tested was a group of paraplegic and tetraplegic patients using a newly developed functional electrical stimulation bicycle system which provided cardiopulmonary reconditioning in addition to muscle strengthening effects. The authors felt the use of computerised closed loop functional electrical stimulation has a definite role in health maintenance prevention of the high morbidity associated with spinal cord injuries. 


\section{Skin Complications in Paediatric/Adolescent Patients with Spinal Cord Injury \\ Paulette M. Harar, M.D., and Puliyodil A. Philip, M.D. \\ Rehabilitation Institute of Chicago, 345 East Superior Street, Chicago, Illinois 60611, U.S.A.}

A prospective study of 20 paediatric/adolescent patients with spinal cord injury seen over a one month period was undertaken and revealed a high incidence of minor skin complications and a low incidence of pressure sores. Three hypotheses were formulated: 1 . The incidence of minor skin complications is greater than the incidence of major complications; 2 . Children less than 15 years of age with spinal cord injury develop minor skin complications; 3. Pressure sores are a developmental issue in teenage males.

A larger study was undertaken in which 64 patients qualified for evaluation between 1 August 1984 and 31 December 1984. In the 16 patients under age 15 , there were five minor skin complications, two second degree burns, two friction problems and one abrasion. In the 45 patients over 15 , none of the females developed a pressure sore, though four had five minor skin complications. Of the 35 males, 26 major and nine minor skin complications were identified. Five of the 14 adolescent males with cervical injuries had six of the skin sores. Fifty percent of the adolescent paraplegic males developed 20 Grade II or greater sores.

It was concluded that our Pediatric Spinal Cord Programme is effective in teaching the parents of our younger children and adolescent females the importance of pressure relief, daily skin inspections and modification of daily routine once a potential sore is identified. It was clear that the adolescent male paraplegic over age 15 years was at high risk for the development of pressure sores. As a result of this study, a prospective longitudinal study looking at other factors is being developed.

\section{Pressure Sore Index}

J. Darrell Shea, M.D., Jose A. Sepulveda, Ph.D., MPH

Humana Hospital-Lucerne Spinal Injury Center, 818 South Main Lane, Orlando, Florida 32801, U.S.A. University of Central Florida, P.O. Box 25000, Orlando, Florida 32816, U.S.A.

One hundred and twenty-one spinal cord injured patients treated at the Lucerne Spinal Injury Center from the onset of injury ('system patient') and sixty patients who came to Lucerne after receiving initial rehabilitation elsewhere ('non-system patients') were evaluated. Patient profiles were similar, e.g., white male, tetraplegic, injured in a motor vehicle accident, average age of 32 . Length of stay (LOS) on readmission was found to be extended by the presence of sores. LOS was significantly longer for non-system patients than system patients. The analysis permitted the development of a profile of the SCI patients at greater risk of developing a pressure sore. This patient is a non-white male, low tetraplegic or high paraplegic, motor and sensory complete from a gunshot wound who is unemployed and who most likely did not complete high school. A pressure sore index, considering the presence, number and grade of pressure 
sores was found to be a useful predictor of clinical course including LOS. This review encourages the recognition of pressure sores as a clinical barometer or index of general SCI patients medical status. The analysis also found that the incidence and severity of pressure sores can be significantly reduced by the development of a SCI patient family education programme.

\section{Performance of Cystograms and Excretory Urograms in Patients with Neurogenic Bladders}

S. L. Stover, M.D., D. M. Witten, M.D., K. V. Kuhlemeier, Ph.D., L. K. Lloyd, M.D., and P. R. Fine, Ph.D.

The University of Alabama at Birmingham, Spain Rehabilitation Center, University Station, Birmingham, Alabama 35294, U.S.A.

Clinical experience in three patients led to a hypothesis that bladder spasms induced by the cystogram examination led to upper urinary tract changes during the excretory urogram. The authors developed this study to examine the effect of performing a cystogram immediately prior to an excretory urogram. Contrast material was injected intravenously for the excretory urogram procedure followed by eight minutes of observation by video monitoring and $\mathrm{x}$-ray films of contrast excretion. In separate studies, iced and room temperature contrast material was introduced by catheter into the bladder to observe its effect on the bladder, ureters, and pelvocaliceal system during excretion of contrast material. Traction on the indwelling catheter was also evaluated during similar procedures. Study population included 16 spinal cord injury patients with neurologically upper or lower motor neuron lesions of at least one year post injury. Some patients were tested more than once. All but one patient were males.

The introduction of iced cystographic contrast material produced increased bladder trabeculation in all nine patients studied with upper motor neuron lesions and in one of the two patients studied with a lower motor neuron lesion. Six of the nine patients with upper motor neuron lesions showed some degree of increased ureterectasis, but none was detected in the two patients with lower motor neuron lesions. Increased pyelocaliectasis was detected in five of nine patients with upper motor neuron lesions; no change in pyelocaliectasis was detected in either patient with a lower motor neuron lesion. The artifactural ureterectasis and pyelocaliectasis did not appear to occur more frequently on either the right or left side.

When room temperature cystographic medium was used, the only changes occurred in one patient with a lower motor neuron lesion who developed mild bladder trabeculation and another patient with an upper motor neuron lesion who developed mild ureterectasis. Catheter traction caused only minimal bladder changes.

Spot $\mathrm{x}$-rays and video-monitoring demonstrated that marked bladder spasm can lead to vesicoureteral obstruction with secondary ureterectasis and pyelocaliectasis. The findings suggest that: 1) a cystogram should not be performed immediately prior to an excretory urogram in patients with neuropathic bladders. If, however, this sequence cannot be avoided because of the need to evaluate for possible vesicoureteral reflux, an adequate recovery period of at least one hour should be allowed between the two procedures; 2 ) the bladder should be 
completely emptied before an excretory urogram is performed; and 3) cystogram contrast material should be at room or body temperature to avoid introducing artificial changes in bladder configuration.

\section{Bladder Distention and Elevation of Body Temperature Following Voiding Cystourethrogram}

Deborah Gaebler, M.D., Jeanne Mervine, R.N., M.S. and Yeongchi Wu, M.D. Rehabilitation Institute of Chicago, Department of Rehabilitation Medicine, Northwestern University-McGraw Medical Center, Chicago, Illinois, U.S.A.

In an effort to demonstrate the relationship between statis and symptomatic bacteriuria in a clinical setting, a consecutive series of 55 traumatic spinal cord injured patients who underwent voiding cystourethrogram and 26 consecutive spinal cord injured patients who underwent urodynamic study were retrospectively reviewed and compared for the presence of post-test fever. The main difference between the two procedures is 1 ) in the voiding cystourethrogram group, the bladder was filled to capacity. This was followed by withdrawal of the catheter for voluntary emptying. If voiding was not possible, the patient was returned to the ward for catheterization after an unknown interval of time. 2) In the urodynamic study, the bladder was filled to the point of reflex detrusor contraction or a maximum of $500 \mathrm{ml}$. The bladder was always emptied at the completion of the test. In both groups bladder distention occurred. Prolonged bladder distension leading to possible upper tract dilatation and stasis occurred only in the patients undergoing voiding cystourethrogram. The patients undergoing urodynamics had temporary bladder distention and were used as control group. The following data was collected: age, date of injury, level of injury, voiding cystourethrogram results, urine culture/senstivity, both pre and post voiding cystourethrogram if available, highest body temperature two days prior to and three days after the test and time interval from voiding cystourethrogram and next catheterization. Patients were included in the study if they had a temperature lower than or equal to 99.1 degrees $F$ preceding the voiding cystourethrogram or urodynamics and were on no antibiotics at the time of the test.

The evidence concluded that information gained from voiding cystourethrogram is useful in managing a neurogenic bladder. However, the complications due to prolonged distention following voiding cystourethrogram for spinal cord injured population has not been recognised. The major premise of this study was that transient bladder distention that occurs sporadically in patients as well as predictably in urodynamics does not contribute to the pathogenesis of symptomatic bacteriuria in the neurogenic bladder. On the other hand, a variable period of bladder distention following the voiding cystourethrogram may be a factor in the pathogenesis of symptomatic bacteriuria in the neurogenic bladder. One possible mechanism suggested for body temperature elevation after voiding cystourethrogram is that prolonged distention of the lower urinary tract leads to upper tract dilatation and stasis with subsequent bacterial involvement of the upper tract. Existence of reflux allows bacteria to freely ascend from the distended bladder. Thus these patients are particularly prone to the consequences of prolonged distention following voiding cystourethrogram. 


\section{Prophylactic Antimicrobial Therapy for Preventing Urinary Tract Infection After Spinal Cord Injury}

K. V. Kuhlemeier, Ph.D., S. L. Stover, M.D. and L. K. Lloyd, M.D.

University of Alabama at Birmingham, Birmingham, Alabama 35294, U.S.A.

The authors conducted the study to determine 1) if any of several antimicrobial agents given in phophylactic doses could reduce the incidence of bacteriuria in SCI patients without indwelling catheters, and 2) if giving these drugs at prophylactic levels resulted in development of bacteria resistant to other drugs.

Two hundred and two males with SCI were evaluated. All had at least one urinary tract infection before being placed on the study. After the urinary tract was rendered sterile by appropriate antibiotic therapy, the subjects were randomly assigned to one of the following drug regimens: A) control-no antimicrobial therapy; B) ascorbic acid, 1 g. q.i.d.; C) trimethoprim/sulphurmethozazole, $80 / 400 \mathrm{mg}$ b.i.d; D) nitrofurantoin macrocrystals, $50 \mathrm{mg}$ t.i.d.; E) nalidixic acid, $500 \mathrm{mg}$ q.i.d.; or F) methenamine hippurate $1 \mathrm{~g}$ b.i.d. Cultures were obtained weekly.

The results suggested that prophylactic antimicrobial therapy is relatively ineffective in preventing bacteriuria in spinal cord injured patients with neuropathic bladders. Ascorbic acid was particularly ineffective. Patients who received ascorbic acid generally had shorter infection-free periods than control patients. Losses of sensitivity to drugs other than the prophylactic drugs were common, especially with carbenicillin.

Although the results of this study suggest that prophylactic treatment for urinary tract infections is relatively ineffective, it must be remembered that the incidence of renal deaths has declined dramatically in recent years. Since this lower death rate has been coincident with a decrease in the use of indwelling catheters and generally vigorous treatment of urinary tract infections, careful study is necessary before a radical change in the urologic treatment of these patients is warranted.

\section{Bacteriuria with Fever After Spinal Cord Injury}

Diana D. Cardenas, M.D. and Michael E. Mayo, M.D.

Departments of Rehabilitation Medicine and Urology, University of Washington, Seattle, Washington 98185, U.S.A.

The authors reviewed an unselected group of 705 spinal cord injury patients treated at the Northwest Regional Spinal Cord Injury Center in an attempt to compare the systems of drainage with the occurrence of bacteriuria with fever. The most common systems of drainage at discharge from the initial hospitalisation for rehabilitation were intermittent catheterisation $(37 \%)$, voiding with or without external collection $(30 \%)$, and use of indwelling catheter $(16 \%)$. Bacteriuria with fever occurred at least once in $43 \%$ of 705 patients during the first hospitalisation and slightly decreased to $30 \%$ of those seen the fourth year follow up. Comparing system of drainage during initial hospitalisation, $42 \%$ of patients with an indwelling catheter experienced bacteriuria with fever; however, $54 \%$ of those on intermittent catheterisation (IC) also experienced bacteriuria with fever. At the first year follow up, $44 \%$ of patients with an indwelling 
catheter, $53 \%$ on self IC, and $83 \%$ of those on IC by someone else reported at least one episode of bacteriuria with fever. Seventy-five patients required sphincterotomy with 58 of those being available for evaluation. In seven of these cases, the sphincterotomy failed. In the remaining 51 patients, $75 \%$ had one or more episodes of bacteriuria with fever during the pre-sphincterotomy period which dropped significantly in the first and fourth year following the surgery.

This analysis would suggest the need for (1) periodic review of the applicability of the IC programme in each individual patient; (2) periodic evaluation of the attendants' of family members' catheterisation techniques in cases where intermittent catheterisation is performed by someone other than the patient; (3) greater patient education regarding sphincterotomy; and (4) consideration of antimicrobial prophylaxis in those subgroups with frequent episodes of bacteriuria with fever.

\section{Suppressive Antibacterial Therapy in the Prevention of Urinary Tract Infection in Spinal Cord Injury Patients}

Linda S. Dean, R.N., F.N.P., M.S.N.

Dallas Rehabilitation Institute, 7850 Brookhollow Road, Dallas, Texas 75235, U.S.A.

Fifteen patients between the ages of 21 and 65 who had been admitted between three and sixty days post injury were selected as subjects for the study. Persons with history of previous renal stones, urinary tract infections or other urological problems prior to their injury were excluded. All patients had normal intravenous pyelograms, had upper motor neuron lesions and had negative urine cultures prior to entry into the study. Patients were on intermittent catheterisation programmes and were catheterised every 4 to 12 hours.

Acceptable subjects were randomly assigned to two groups. One received no prophylactic antibacterial treatment and the second group received on altering months Trimethoprim $80 \mathrm{mg}$, Sulphurmethoxazol $400 \mathrm{mg} 1$ p.o. b.i.d., and Nitrofurantoin Macrocrystals $50 \mathrm{mg} 1$ p.o. b.i.d. Ascorbic acid supplements were not administered to either group. Daily $\mathrm{pH}$ and dip-slide uricult was obtained on each subject. Bacterial growth was reported after 24 hours of incubation and a urine culture was obtained. Culture growth grew greater than 10000 colonies was definitive criteria for urinary tract infection. Those found to have urine infections were treated with appropriate antibiotics for seven days.

The results revealed 18 urinary tract infections were present in group I with a total cost of treatment at $\$ 476.00$. Group II had a total of 17 urinary tract infections with a total cost of treatment at $\$ 6,717.00$. No significant statistical difference was seen for the number of UTI's using an independent T-test for difference of two variables, $\mathrm{p}=.48$. 


\section{Urinary Collagen Metabolite Concentration as a Predictor of Compli- cations in Spinal Cord Injury}

Gladys P. Rodriguez, M.S., Jacqueline Claus-Walker, Ph.D., Mary C. Kent, B.S. and R. E. Carter, M.D.

Department of Rehabilitation, Baylor College of Medicine, The Institute for Rehabilitation and Research, Houston, Texas, U.S.A.

Immediately after the trauma, spinal cord injury patients have increased rate of collagen synthesis and an even greater increase in collagen degradation. The loss of collagen from bone, accompanying a loss of calcium, is implicated in the aetiology of urinary tract stones, heterotopic ossification, and osteoporosis; similarly, the loss of skin collagen might lead to a propensity to develop skin ulcers.

To study the relationship between collagen degradation and the incidence of bone or skin related complications in spinal cord injury, two collagen metabolites, glucosyl-galactosyl hydroxylysine and galactosyl hydroxylysine were measured. Controls were developed from individuals with no known chronic disease. Urine samples were collected at appropriate intervals during hospitalisation and then on outpatient clinic visits. Studies indicated that an increase in the glucosylgalactosyl hydroxylysine excretion precedes the appearance of overt symptoms of skin inflammation by about one month. We cannot, at this time, demonstrate a similar temporal relationship between an increase in urinary galactosyl hydroxylysine excretion and genesis of bone related complications.

Being able to predict the appearance of bone or skin related complications would enable the physician to initiate appropriate treatment which might alleviate or prevent the problem.

\section{Symbiotic Regression of Adolescent Boys and Their Mothers, A Complication of High Spinal Cord Injuries}

Kenneth H. Gordon, M.D., M.S. (Psychiatry)

Spinal Cord Injury Unit, Shriners Hospital for Crippled Children, Thomas Fefferson University, Philadelphia, Pennsylvania, U.S.A.

Of one hundred and six patients treated for spinal cord injury, forty-two boys were tetraplegic and thirty-six were paraplegic. Thirteen girls had tetraplegia quadriplegia and fifteen were paraplegic. Five adolescent boys developed a peculiar regression with their mothers which interrupted their rehabilitation programme. Each of these five young men had been his mother's 'golden one' before the accident with their mothers tending to live through these sons, counting on the boys' future accomplishments to provide their own fulfillment as persons. In each case, both mother and son were unable to accept the injury which resulted in denial and regression together. No patients with injuries lower than $\mathrm{T}-2$, no boys who were not adolescents, and no girls developed this syndrome.

Characteristics of symbiotic regression are 1) isolation from hospital staff and seclusion of the couple in a darkened room; 2) blurring of boundaries between mother and son; 3) the adolescent boy regresses to act like a three or four month old infant; 4) blurring of boundaries between mother and hospital staff; 5) 
paranoid distrust of hospital staff first manifested by refusal to eat or drink hospital food; 6) promise of law suits and threats to sign out against medical advice; 7) manipulation of the staff by the couple leads to hostile relations between staff members who previously worked well together; 8) staff becomes afraid of the mother. Treatment and prevention consisted of removing the adolescent from the darkened room, placing him in the company of peers during daytime and staff at night, e.g., adolescent to sleep at nursing station; each member of the staff to spend $30+$ minutes each day talking with previously feared mother; encourage the mother and boy to be separate independent human beings. No formal psychiatric treatment is required.

With this simple treatment, symbiotic regression disappeared within two days and since our last case we have been able to prevent it.

\section{Prevention of Pulmonary Complications in Spinal Cord Injury with the Rotorest Bed}

Robert Harris, M.D., H. David Reines, M.D.

Department of Anaesthesiology and Surgery, Medical University of South Carolina, 171 Ashley Avenue, Charleston, South Carolina 29425, U.S.A.

Pulmonary complications secondary to immobilisation and decreasing vital capacity account for $50 \%$ of deaths in patients with spinal cord injuries. This study was performed to evaluate the effectiveness of a kinetic rotobed therapy in the prevention and treatment of pulmonary complications in spinal cord injured patients.

A retrospective study previously reported 103 patients treated 1975-79 were labelled group I. A second group of patients treated from 1980-84 was labelled group II and was treated similar to group I except for the addition of the rotating bed. Complications evaluated included atelectasis, pulmonary oedema and pneumonia, pulmonary emboli, aspiration syndrome and death. The results are presented in the table.

Results

\begin{tabular}{|c|c|c|c|c|c|c|c|c|}
\hline \multirow{3}{*}{$\begin{array}{l}\text { Gp. I } \\
\text { Gp. II }\end{array}$} & \multicolumn{2}{|c|}{ Atelectasis } & \multicolumn{2}{|c|}{$\begin{array}{c}\text { Pulmonary } \\
\text { oedema }\end{array}$} & \multicolumn{2}{|c|}{$\begin{array}{l}\text { Pulmonary } \\
\text { emboli }\end{array}$} & \multicolumn{2}{|c|}{ Aspiration } \\
\hline & 17 & $16 \%$ & 4 & $4 \%$ & 4 & $4 \%$ & 6 & $6 \%$ \\
\hline & 1 & $5^{\circ}$ & 0 & 0 & 0 & 0 & 0 & 0 \\
\hline \multicolumn{3}{|c|}{ Pneumonia } & \multicolumn{2}{|c|}{ Death } & & & & \\
\hline Gp. I & 11 & $11 \%$ & 17 & $16 \%$ & & & & \\
\hline Gp. II & 2 & $10 \%$ & 8 & $40 \%$ & & & & \\
\hline
\end{tabular}

Analysis of the eight deaths in Group II revealed that four died with pulmonary complications which developed or progressed after rototherapy was discontinued. The remaining four died of complications which are present prior to placing the patient on the bed. Therefore, the high mortality rate in Group II reflects a subset of patients who were (a) at high risk for pulmonary death, and (b) patients no longer treated on the rotorest bed. The authors suggest their findings indicated patients at high risk for developing pulmonary complications associated with spinal cord injury may benefit from early kinetic therapy. 


\section{Tracheotomy following Spinal Cord Injury}

M. Hugh Bailey, M.D., Ch.B., M. Christine Stock, M.D. and Victor L. Lewis, Jr., M.D.

Northwestern University, 251 East Chicago Avenue, Chicago, Illinois 60611, U.S.A.

Patients who had tracheotomy performed at the spinal cord injury unit at Northwestern university for five years were reviewed. All these were done under the direct supervision of Victor L. Lewis using a standardised technique. The tracheotomy was usually performed through the third tracheal ring but occasionally the second or fourth was utilised. There were no serious intraoperative complications with blood loss typically less than $5 \mathrm{cc}$. Early postoperative complications such as infection, airway obstruction, haemorrhage, erosion of adjacent structures or subcutaneous emphysema were rare. There was no tracheostomy related mortality. Late postoperative complications including tracheal stenosis were rare, although many of the patients had their tracheotomies for many months.

The authors conclude that tracheotomy is an integral part of the successful management of patients with cervical spine injuries. When performed under the described conditions, using a standardised surgical method, protocol of tracheotomy care, and ventilatory support, it is a safe procedure, with a low risk of significant complications. The benefits include prevention and treatment of pulmonary complications, assistance in weaning the patient from mechanical ventilation, and patient comfort. Early patient selection and relatively liberal indications are warranted. Reduction in morbidity and mortality from pulmonary complications will result.

\section{Progressive Post-traumatic Cystic Myelopathy: A Four Year 60 Patient Experience}

Barth A. Green, M.D., F. J. Eismont, M.D., R. M. Quencer, M.D., B. Montalvo, M.D. and J. Post, M.D.

University of Miami School of Medicine, 1501 Northwest 9th Avenue, Miami, Florida 33136, U.S.A.

Over a period of four years, 60 cases of post-traumatic spinal cord cysts were identified, and 42 of these patients received surgical treatment. Presenting signs and symptoms appeared from three months to twenty years following trauma with one or more of the following: progressive loss of motor and/or sensory function, local and/or radicular pain, increasing spasticity, dysreflexia, sweating, posturally related respiratory distress, and an alternating Horner's syndrome. Diagnosis was made with delayed metrizamide CT scanning with the Magnetic Resonance Investigators more recently providing a non-invasive alternative.

Treatment involved both a cyst to subarachnoid space shunt using a silastic catheter, and an untethering of the spinal cord or nerve root adhesions. Surgery was only performed when progressive functional loss was demonstrated. Recent surgical technical advances include the use of intraoperative spinal evoked potential monitoring, microlaser myelotomy and realtime ultrasonography. The best surgical results were noted in patients with loss of motor and/or sensory function with the least success in patients operated on for reduction of spasticity. Compli- 
cations included a loss of some degree of dorsal column sensation postoperatively in 10 percent and a ten percent re-exploration rate. It was felt that the progression of these cystic lesions and resulting symptomatology is associated with shearing forces associated with local tethering of the spinal cord and/or nerve roots at the lesion site.

\section{Spinal Cord Injury in Patients with Ankylosing Spondylitis}

Allen P. McDonald, M.D.

Shepherd Spinal Center, 2020 Peachtree Road, N.W., Atlanta, Georgia 30309, U.S.A.

The author reviewed experiences with five patients with ankylosing spondylitis who had significant trauma when involved in either automobile accidents or motorcycle injuries. All patients had known ankylosing spondylitis for many years prior to spinal cord injury.

The keys to management are reduction of the fracture by appropriate traction which must be applied in such a way to immobilise the patient in the cervical attitude of preinjury state. Stabilisation must be maintained for sufficient length of time to allow bony healing. These principles are the same as management of any fracture. Generally in the cervical spine, the halo ring should be applied initially in the Emergency Room and reduction maintained in over the bed traction. Following medical stabilisation of the patient and adequate $\mathrm{x}$-rays documenting reduction of the fracture, then the halo vest can be applied.

All the patients managed in this way healed with no significant residual deformity. No patients died.

The authors concluded that if careful attention is applied to restoring the premorbid cervical state and maintaining this traction even though it requires imaginative approaches, the patient with rather marked deformities can be managed satisfactorily without undue morbidity.

\section{Hip Joint Changes in Spinal Cord Injured Patients}

Rafael D'Auria, M.D., Chi-Tsou Huang, M.D., Samuel L. Stover, M.D. and Philip R. Fine, Ph.D.

Department of Rehabilitation Medicine, The University of Alabama at Birmingham, Spain Rehabilitation Center, University Station/Birmingham, Alabama 35294, U.S.A.

A retrospective review of medical records of spinal cord injured patients treated at the University of Alabama at the Spain Rehabilitation Center identified seven patients with progressive hip changes. Six were male. Two were involved in automobile accidents, two motorcycle accidents, one gunshot wound, one diving and one waterskiing. All patients had a neurologically complete spinal cord lesion with moderate to severe spasticity. Four patients had evidence of heterotopic ossification. There was no history of any of hip trauma.

Review of the clinical histories did not give an indication of the underlying aetiology of pathogenesis of these changes. It was suggested that the possibility of autonomic nervous system dysfunction may have a role in the pathogenesis of these hip joint changes. All these patients had levels above the sixth thoracic 
segment with histories of multiple episodes of autonomic hyperreflexia. It was noted that once hip joint changes occurred, they rapidly progressed suggesting that prevention should be the aim rather than treatment. Preventive measures worth considering are close monitoring of the hip status to detect any changes as early as possible. Once these have been detected then release of the hip by multiple tenotomies should be considered.

\section{Energy Cost of Paraplegic Ambulation}

Robert L. Waters, M.D. and Brenda Rae Lunsford, R.P.T.

Rancho Los Amigos Hospital, 7601 East Imperial Highway, Downey, California 90242, U.S.A.

Physiological energy expenditure associated with several modes of mobility was measured in 151 paraplegic spinal injured patients. High level paraplegics with extensive paralysis requiring bilateral knee-ankle-foot orthoses to walk with a swing-through crutch assisted gait had an average rate of oxygen consumption of $16.4 \mathrm{ml} / \mathrm{kg} / \mathrm{minute}$ which was $43 \%$ greater than for wheelchair propulsion (11.5 $\mathrm{ml} / \mathrm{kg} /$ minute). Their mean walking speed was slow, $29 \mathrm{~m} /$ minute in comparison to wheelchair propulsion of $72 \mathrm{~m} /$ minute or normal walking of $80 \mathrm{~m} /$ minute. Furthermore, even low lumbar paraplegics with bilaterally intact hip flexor and knee extensor muscles who were able to walk with a reciprocal crutch assisted gait not requiring knee-ankle-foot orthoses had elevated rate of oxygen consumption averaging $13.8 \mathrm{ml} / \mathrm{kg} /$ minute which was $20 \%$ greater than for wheeling and $15 \%$ greater than for normal walking. In addition, their mean walking speed was the slowest of all the groups, $26 \mathrm{~m} /$ minute.

It is felt that these findings accounted for the common clinical experience that most high level paraplegics who required bilateral knee-ankle-foot orthosis and utilise a swing-through crutch assisted gait prefer wheeling and discontinue walking as the primary means of ambulation following gait training. 